\title{
邻氨基芳香腈与羰基化合物的反应机理及其产物的骨架结构
}

\author{
杨俊娟 ${ }^{a}$ 史大昕 ${ }^{a}$ 刘明星 ${ }^{a}$ 张立军 ${ }^{b}$ 张 奇 ${ }^{a}$ 李加荣 $*, a$ \\ ${ }^{a}{ }^{a}$ 北京理工大学化工与环境学院 北京 100081) \\ ${ }^{b}$ 天津理工大学化学化工学院 天津 300191$)$
}

\begin{abstract}
摘要 Friedländer 反应是合成具有生物活性、光电活性的喹啉衍生物的重要方法. 其典型转化之一一一邻氨基芳香腈 与羰基化合物的 Friedländer 缩合在得到经典的转化产物的同时可以得到新骨架产物, 这种新转化自本课题组报道以来 已受到国内外学者的诸多关注, 但该转化的新产物骨架结构尚存争议. 本综述概括了该新转化自发现以来国内外的研 究进展, 从 ${ }^{13} \mathrm{C}$ NMR, FT-IR, X 射线单晶衍射数据等明确了新转化反应得到的产物骨架为喹唑啉酮结构. 完整地提出了 该类 Friedländer 分岔反应的转化机理: 经由分子内的 Pinner 反应到 Dimroth 重排的过程存在于邻氨基芳香腈和酮的经 典 Friedländer 反应中(PDF 反应).
\end{abstract}

关键词＼cjkstart邻氨基芳香腈; 分忿转化; Friedländer 转化; 喹唑啉酮; 苯并噁嗪; 产物结构

\section{Structure of the Condensed Product of Aromatic o-Aminonitrile with Carbonyl Compound and Its Mechanism}

\author{
Yang, Junjuan ${ }^{a}$ \\ Shi, Daxin ${ }^{a}$ \\ Liu, Mingxing ${ }^{a}$ \\ Zhang, Lijun ${ }^{b}$ \\ Zhang, $\mathrm{Qi}^{a}$ \\ Li, Jiarong*,a \\ ( ${ }^{a}$ School of Chemical Engineering and Environment, Beijing Institute of Technology, Beijing 100081) \\ ( ${ }^{b}$ School of Chemistry and Chemical Engineering, Tianjin University of Technology, Tianjin 300191)
}

\begin{abstract}
Friedländer reaction is one of the most important routes to synthesize the quinoline and its derivatives, which possesses excellent bioactivity and photo-electricity activity. Recently, a new skeleton product besides the normal Friedländer quinoline was discovered by the condensation of aromatic $o$-aminonitrile and carbonyl compounds, and this phenomenon has attracted many researchers, but the skeleton structure of new conversion is debated. Herein, this paper reviews on the development progress of this new kind reaction. According to recent research results, the skeleton structure of new conversion was assigned as quinazolinone, which was confirmed by the ${ }^{13} \mathrm{C}$ NMR, FT-IR, and single-crystal X-ray diffraction deterimations. Therefore, this new conversion is abbreviated as PDF conversion, which means a new conversion from Pinner to Dimroth rearrangement in the Friedländer reaction. The total reaction mechanism of $o$-aminonitrile with carbonyl compound was proposed.
\end{abstract}

Keywords aromatic $o$-aminonitrile; divergent conversion; Friedländer conversion; quinazolinone; benzoxazine; product structure

邻氨基芳香腈是一类非常有用的双官能团化合物, 相邻的氨基、氧基的推、拉电子效应赋予整个体系特有 的化学性质, 由此可以合成一系列含氮杂环化合物 ${ }^{[17]}$. 这类物质的典型转化是它与羰基底物经由 Friedländer 缩合制备 4-氨基吡啶及其相关衍生物 ${ }^{[8-10]}$. 有意思的是 当我们用 2-氨基-5-硝基苯甲腈(1a)与环己酮(2a)在氯化 锌催化下回流制备抗老年痴呆药物他克林的 7-硝基衍
生物时，除了得到预期的目标物 3a 外，还得到一种浅黄 色的固体 ${ }^{[11]}$. 当我们改变底物结构时, 依然可以得到除 预期目标物外的相似结构的浅黄色固体. 这类新产物的 骨架结构早期被认为是 $2 H-3,1$-苯并噁嗪(4) (图 1), 但随 后我们将该物质的骨架结构调整为喹唑啉酮 $\mathbf{5}^{[12 \sim 22]}$.

这是国内外首次发现这类 Friedländer 反应在生成 正常喹啉衍生物的同时还能产生不同骨架的新产物, 这

*E-mail: jrli@bit.edu.cn

Received June 6, 2014; revised July 16, 2014; published online August 26, 2014

Project supported by the Basic Research Fund of Beijing Institute of Technology (No. 2012CX100035).

北京理工大学基础研究基金(No. 2012CX100035)资助项目. 


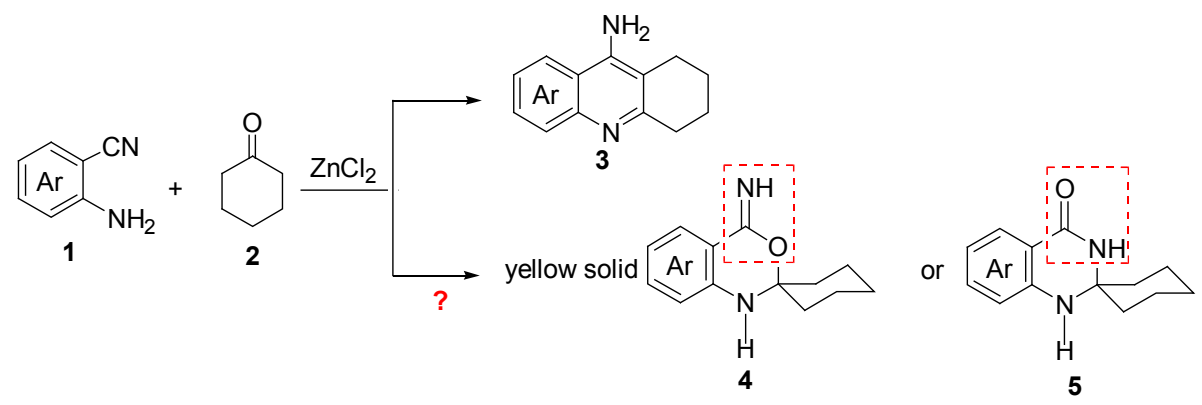

图 1 邻氨基芳香腈与环己酮的反应

Figure 1 Reaction of aromatic $o$-aminonitrile and cyclohexanone

种现象也在随后的研究中为国内外的多个课题组所证

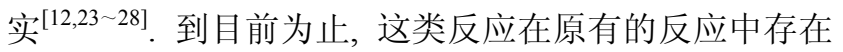
可以得到新骨架产物的转化已成不争的事实, 不能统一 的是新转化产物的骨架结构到底是 3,1-苯并噁嗪(4)还 是喹唑啉酮 5 .

为了弄清这个问题则需要了解这个反应的转化机 理. 结合经典的邻氨基芳香醛与含有 $\alpha$-活泼氢的羰基化 合物反应生成喹啉衍生物的机理 ${ }^{[29-35]}$, 一般认为, 此类 邻氨基芳香腈与羰基化合物缩合首先是得到羟胺加成 物 6, 6 失水得到 Shiff 碱 7, 7 重排成 8 后发生分子内的 关环得到 9, 9 异构化得到正常的 Friedländer 产物 3. 那 么新的转化极有可能是羟胺中间体 6 不失水而直接进行 分子内反应, 即着基进攻氰基得到噁溙衍生物 4(图 2).

由图 1 所示的反应过程为例, 2-氨基-5-硝基苯甲腈 (1a) 与环己酮 $2 \mathrm{a}$ 新转化的产物可能是下列物质之一: 预 期目标物一一喹啉结晶水合物 $\mathbf{3 a}$, 氨基进攻羰基所得
的着弪胺中间体 $\mathbf{6 a}$, 该中间体失水所得的 Shiff 碱 7a, $\mathbf{6 a}$ 的羟基进攻氰基形成的亚胺噁嗪 $4 a$ 和 $4 a$ 的同分异构体 5a(图 3). 其中前三者可以很容易从产物的 IR、NMR 和 $\mathrm{MS}$ 表征得到排除, 所剩的结构只能是 $\mathbf{4 a}$ 和 $\mathbf{5} \mathbf{a}^{[11]}$. 但由 于亚胺苯并噁嗪 $4 \mathbf{a}$ 和喹唑啉䣶 $5 \mathbf{a}$ 的结构差别只是环 3和 4-位侧链双键端基的 $\mathrm{O}$ 原子及 $\mathrm{NH}$ 基团之间发生了互 换，两者的元素分析、红外和质谱数据几乎没有差别， 核磁能够给出细小的差别, 很难判断, 因此才会有近年 来关于此类反应新转化产物结构的争论.

我们早期认为新转化产物的骨架结构是噁嗪 $\mathbf{4}^{[11]}$, 西班牙学者 León 等 ${ }^{[36]}$ 也在相应的研究中得到了类似的 结果(Eq. 1). León 等使用邻氨基氰基吡喃 $\mathbf{1 0}$ 与环已酩在 $\mathrm{AlCl}_{3}$ 催化下反应 $14 \mathrm{~h}$ 以上, 除了得到正常的 Friedländer缩合产物 $\mathbf{1 1}$ 之外，也获得了噁溙产物 12 (Eq. 1). 2010 年. 印度学者 Jachak 等 ${ }^{[37]}$ 也报道了 2-氨基-3-氰

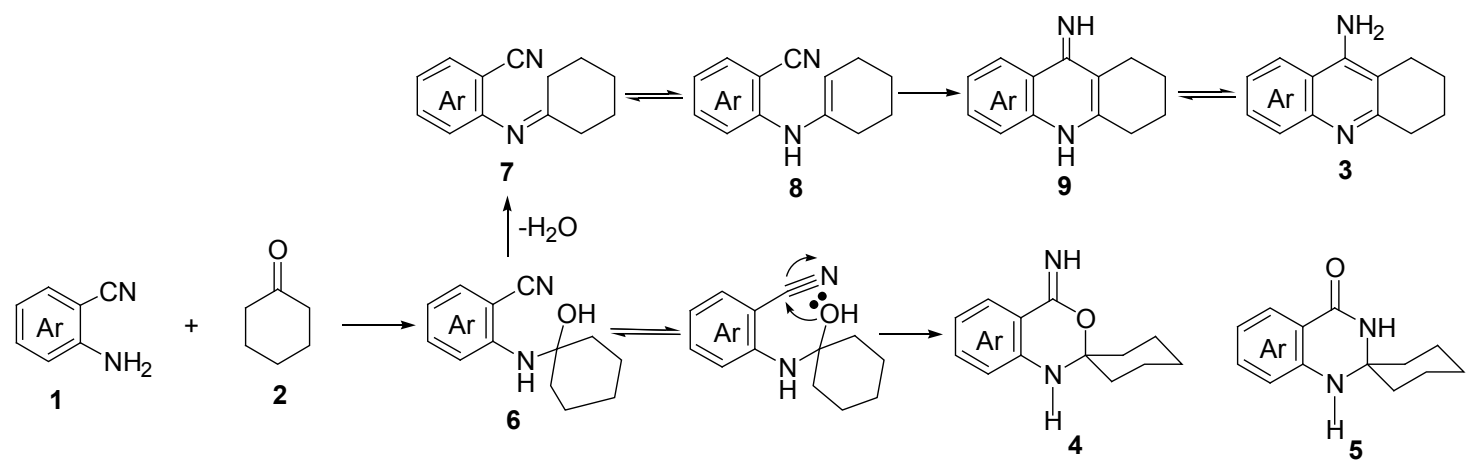

图 2 邻氨基芳香腈与环己酮的一般反应过程

Figure 2 Possible reaction mechanism of aromatic $o$-aminonitrile and cyclohexanone<smiles>Nc1c2c(nc3ccc([N+](=O)[O-])cc13)CCCC2</smiles>

3a<smiles>N#Cc1cc([N+](=O)[O-])ccc1NC1(O)CCCCC1</smiles>

$6 a$<smiles>Cc1cc([N+](=O)[O-])ccc1N=C1CCCCC1</smiles>

4a<smiles>O=C1NC2(CCCC2)Nc2ccc([N+](=O)[O-])cc2C1=O</smiles>

$5 a$

图 3 2-氨基-5-硝基苯甲腈与环己酮反应的可能产物

Figure 3 The proposed structure for the reaction of 2-amino-5-nitrobenzonitrile and cyclohexanone 
基喹啉(13)与环己酮及其衍生物 14 在 $\mathrm{ZnCl}_{2}$ 催化下, 得 到的产物为螺[恶嗪并[4,5- $b$ ] 喹啉-2,1'-环己烷]-4(1H)-亚 胺结构产物 15 (Eq. 2).
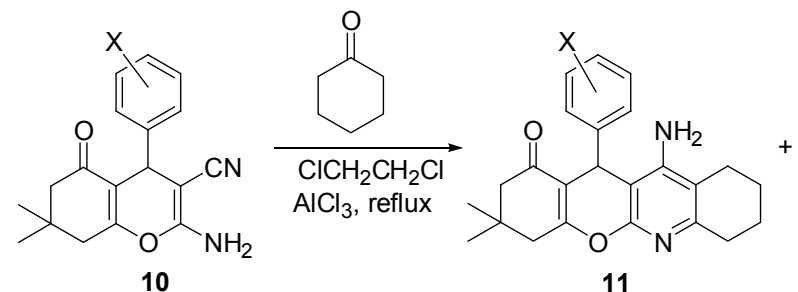<smiles>[X]c1ccc(C2OC3(CCCCC3)OC3=C2C(=O)CC(C)(C)C3)cc1</smiles><smiles>N#Cc1cc2ccccc2nc1N</smiles>

13<smiles>[R]C1CCC(=O)C([R])C1[R]</smiles>

\section{$\underset{\mathrm{ZMF}, \text { reflux }}{\stackrel{\mathrm{ZnCl}}{2}}$}

14<smiles>[R]C1CCC2(Nc3ccccc31)Nc1nc3ccccc3cc1C(=N)O2</smiles>

2008 年, 在对比新转化产物与已知不同骨架化合 物的核磁图谱及其单晶衍射数据后, 我们 ${ }^{[22]}$ 将新产物 的骨架结构由噁溙 4 调整为喹唑啉酮 5. 捷克学者 Čejka 等 ${ }^{[38]}$ 于 2012 年的相应研究结果也表明其转化产物的骨 架结构为喹唑啉酮: 在 $\left[\mathrm{CU}_{3}(\mathrm{BTC})_{2}\right]$ 催化下，4-氯-2-氨基 苯甲腈 $(\mathbf{b})$ 与环己酮反应, 得到了喹啉和喹唑啉酮两种 衍生物 $3 \mathrm{~b}$ 和 $\mathbf{5 b}$, 两者的比例分别为 $58 \%$ 与 $42 \%$ (Eq. 3).<smiles>N#Cc1ccc(Cl)cc1N</smiles>

天津理工大学张立军等 ${ }^{[39]}$ 采用杂均相催化技术也 成功实施了邻氨基芳香腈与羰基化合物缩合生成喹唑 啉䣶衍生物的转化. 尤其是近期浙江科技大学 $\mathrm{Wu}$ 和德 国 Rostock 大学的 Langer 等 ${ }^{[23]}$ 合作研究了磷酸钾催化、
水相中邻氨基芳香腈与醛的反应，其产物骨架结构用 2D 核磁技术证明是喹唑啉酮 $\mathbf{5}$.

本文全面回顾近年来有关此类 Friedlander 转化新 产物骨架结构是喹唑啉酮 $\mathbf{5}$ 的证据.

\section{$1{ }^{13}$ C NMR 化学位移}

在一维核磁中, 亚胺噁嗪和喹唑啉酮的 NH 质子氢 谱的化学位移差异不大，很难区分; 而两者的相应碳原 子由于所处环境不同，因此碳谱存在可以区分的差异. 图 4 列出了一些已知噁嗪和喹唑啉酮化合物的 ${ }^{13} \mathrm{C} \mathrm{NMR}$ 特征数据 ${ }^{[40 \sim 47]}$. 由图 4 可以看出, 稠合杂环的 $N, O$ 原子 换位以后，对应的 ${ }^{13} \mathrm{C}$ 波谱的化学位移出现一定的区别, 主要体现在 2-位碳的化学位移由亚胺噁嗪的 $\delta 75$ 降到 喹唑啉酩的 $\delta 63 \sim 68$, 而 4-位碳的化学位移从相应的 $\delta$ 147 变为 $161 \sim 164$.

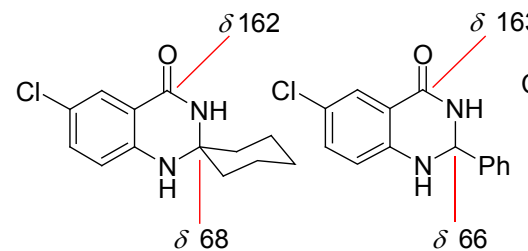<smiles>CC(C)(C)[C@H]1Nc2ccc(Cl)cc2C(=O)N1c1ccccc1</smiles><smiles>C=CCC1N([13CH3])C(=O)c2cc(Cl)ccc2N1CC=C</smiles><smiles>CC1Nc2cc3c(cc2C(C)(C)O1)C(C)(C)OC(C)N3</smiles><smiles>[R]NC(C)c1nc2ccccc2c(=NC([R])C(C)=O)o1</smiles>

$\delta 161.7$<smiles>Cc1nc2ccccc2c(=O)n1C</smiles>

图 4 一些已知化合物的核磁碳谱特征数据

Figure 4 The ${ }^{13} \mathrm{C}$ NMR data of some known compounds

图 5 给出了 Klemm 等 ${ }^{[48]}$ 于 1998 年用邻氨基苯甲酰 胺和环戊酮为原料合成所得的喹唑啉酮和我们课题 组 ${ }^{[49]}$ 用邻氨基苯甲腈和环戊酮为原料合成 Friedlander 反应生成副产物的碳谱数据. 从图 5 中可以看出, 其碳 谱数据基本一致，其 2-位碳的 $\delta$ 值均为 77.1, 4-位碳的 $\delta$ 值为分别为 $\delta 163.4$ 和 163.5 , 因此可以说明此类 Friedlander 转化新产物骨架结构是喹唑啉酩.

更有说服力的则是, Rostock 大学的 Langer 等 ${ }^{[23]}$ 成 功地用 2D 核磁技术(H-H-COSY 谱)研究了新转化产物 的骨架结构(图 6). 结果表明: 该化合物的质子有两组 自旋偶合常数, 分别为喹唑啉酮的两种环 $\mathrm{N}-\mathrm{H}$ 质子与 苯环邻位质子 $(\mathrm{Ph}-\mathrm{H})$ 远程作用所致; 这种相互作用也 为 NOESY 测试结果所证实. 此现象只有喹唑啉酮的骨 

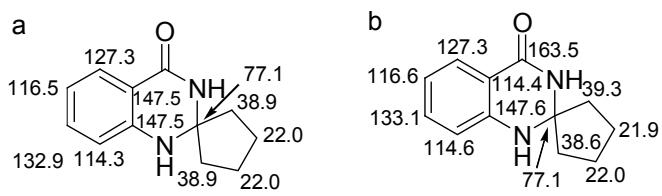

图 5 本课题组(a)和 Klemm 课题组(b)报道的喹唑啉酮核磁碳 谱数据

Figure 5 The ${ }^{13} \mathrm{C}$ NMR data reported by both us (a) and Klemm team (b)

架结构才能满足, 而啞嗪结构则不能.

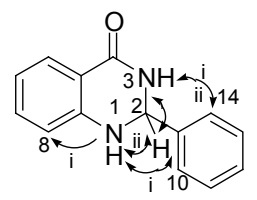

图 6 化合物 2-芐基-2,3-二氢喹唑啉酮

Figure 6 2D-NMR-coupling pattern of 2-phenyl-2,3-dihydroquinazolin-4(1H)-one

\section{2 单晶测定}

尽管单晶测定是有机化合物结构确定的最直接和 有效方法, 遗憾的是这种新转化产物的可能结构差别十 分微细，单晶测定后对其结构用不同的骨架类型进行解 析所得结果差别不大, 由此造成我们早期将新转化产物 确定为前者而不是后者. 为了准确确定新转化产物的结 构, 同时为了便于和噁嗪骨架结构化合物进行比较, 我 们 ${ }^{[22,50 ~ 54]}$ 在制得了邻氨基苯甲腈 $1 \mathrm{c}$ 与环戊酮的新转化 产物二氢喹唑啉酮 16 的单晶并进行测定的基础上(图 7, 表 $1 \sim 3$ ), 又专门用邻氨基苯甲酸(17)与环酮反应(图

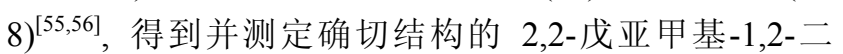
氢-3,1-苯并噁嗪-4(3H)-酩 (18)的单晶数据(图 7, 8, 表 $4 \sim 6$ ).

对比这两个晶体的结构参数发现, 化合物 16 的 $\mathrm{N}(1)-\mathrm{C}(8)$ 和 $\mathrm{N}(2)-\mathrm{C}(8)$ 键长比较接近, 分别为 $0.14661(14)$ 和 $0.14534(14) \mathrm{nm}$; 而噁嗪 18 的 $\mathrm{O}(1)-\mathrm{C}(8)$ 和 $\mathrm{N}(1)-\mathrm{C}(8)$ 键长分别为 $0.1469(2)$ 和 $0.1438(2) \mathrm{nm}$, 两 键长相差较大一些, 符合 $\mathrm{O}, \mathrm{N}$ 原子差异. 另外, $\mathrm{O}(1)$ 或 $\mathrm{N}(1)$ 在环内时, 由于原子半径和成键能力的差异, 导致 含双杂原子的六元环具有不同的形变, 这一点也可从两 者的扭角数据中看出.

结合晶胞堆积图 9 及结构参数可知, 啞嗪分子间形 成单一氢键结构片段, 键角为 $160.3(17)^{\circ}$, 说明分子间 氢键较弱, 这与分子的稳定性相关(噁嗪是一种不太稳 定的分子), 另外, 也和其较低的熔点数据(143.4 145 $\left.{ }^{\circ} \mathrm{C}\right)$ 相符合. 而由邻氨基苯甲腈得到的新转化产物 16 分子间形成一种典型的 $\mathrm{R} 22(8)$ 氢键 ${ }^{[57,58]}$, 键角为 $171.4(14)^{\circ}, 173.0(15)^{\circ}$, 较噁嗪分子更接近于 $180^{\circ}$, 这是

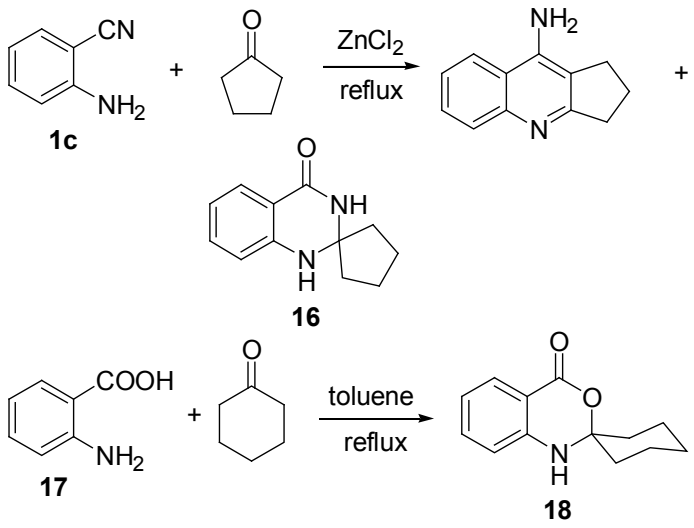

图 7 邻氨基苯甲腈和邻氨基苯甲酸分别与环酮的反应

Figure 7 Reaction of cyclohexanone $o$-aminobenzamide or/and $o$-aminobenzoic acid
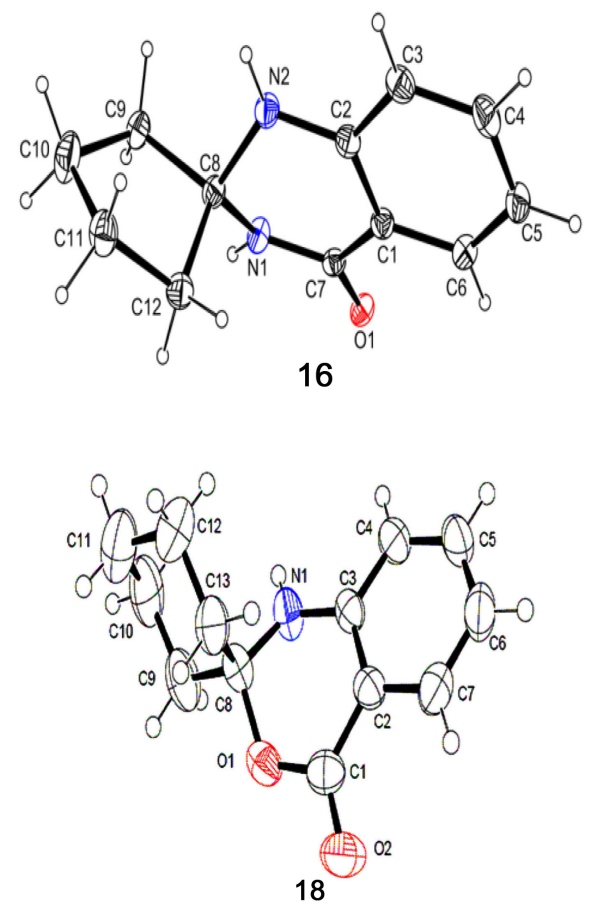

图 8 化合物 16 和 18 的晶体结构图(50\%椭球体)

Figure 8 X-ray crystallographic of $\mathbf{1 6}$ and $\mathbf{1 8}$

一种较强的氢键形式, 化合物稳定性较高, 其熔点高达 $268 \sim 270{ }^{\circ} \mathrm{C}$; 当我们用 ChemBioDraw 3D 软件对化合 物 16 的喹唑啉酮结构进行解析, 喹唑啉酮的总能量为 $35.4 \mathrm{~kJ} / \mathrm{mol}$, 如果调整为噁嗪结构再进行模拟, 其总能 量为 $81.5 \mathrm{~kJ} / \mathrm{mol}$. 由此可知: 前者结构在热力学上更为 稳定, 这也说明新转化产物的结构应为喹唑啉酩. 另外, 环外的羰基氧原子同时与两个分子成氢键作用，此位换 为亚胺基团是不合理的. 更重要的是, $R$ 因子和 $S$ 因子等 重要参数也指示了喹唑啉酮模型的正确性. 即当我们把 化合物 16 的结构解析为亚胺苯并噁嗪结构时 ${ }^{[59,60]}$, 其 最终残差因子 $R_{1}=0.0994, w R_{2}=0.3001$, 相比于喹唑啉 
表 1 化合物 16 的特征键长和键角

Table 1 Bond lengths and angles for compound 16

\begin{tabular}{rl||cl}
\hline Bond & Length/nm & Bond & Angle $/\left({ }^{\circ}\right)$ \\
\hline $\mathrm{N}(1)-\mathrm{C}(8)$ & $0.14661(14)$ & $\mathrm{C}(2)-\mathrm{C}(1)-\mathrm{C}(7)$ & $118.34(10)$ \\
$\mathrm{N}(1)-\mathrm{H}(1)$ & $0.0884(16)$ & $\mathrm{N}(2)-\mathrm{C}(2)-\mathrm{C}(1)$ & $119.33(10)$ \\
$\mathrm{N}(2)-\mathrm{C}(2)$ & $0.13712(14)$ & $\mathrm{N}(1)-\mathrm{C}(7)-\mathrm{C}(1)$ & $116.04(9)$ \\
$\mathrm{N}(2)-\mathrm{C}(8)$ & $0.14534(14)$ & $\mathrm{N}(2)-\mathrm{C}(8)-\mathrm{N}(1)$ & $107.03(9)$ \\
$\mathrm{N}(2)-\mathrm{H}(2)$ & $0.0888(17)$ & $\mathrm{N}(2)-\mathrm{C}(8)-\mathrm{C}(9)$ & $110.00(9)$ \\
\hline
\end{tabular}

表 2 化合物 16 的特征扭角

Table 2 Angles for compound 16

\begin{tabular}{cc||cr}
\hline Bond & Angle $/\left(^{\circ}\right)$ & Bond & Angle $/\left({ }^{\circ}\right)$ \\
\hline $\mathrm{C}(8)-\mathrm{N}(2)-\mathrm{C}(2)-\mathrm{C}(3)$ & $-158.11(10)$ & $\mathrm{C}(8)-\mathrm{N}(1)-\mathrm{C}(7)-\mathrm{O}(1)$ & $169.91(10)$ \\
$\mathrm{C}(8)-\mathrm{N}(2)-\mathrm{C}(2)-\mathrm{C}(1)$ & $25.54(15)$ & $\mathrm{C}(6)-\mathrm{C}(1)-\mathrm{C}(7)-\mathrm{O}(1)$ & $-6.68(16)$ \\
$\mathrm{C}(6)-\mathrm{C}(1)-\mathrm{C}(2)-\mathrm{N}(2)$ & $177.12(10)$ & $\mathrm{C}(2)-\mathrm{C}(1)-\mathrm{C}(7)-\mathrm{O}(1)$ & $165.62(10)$ \\
$\mathrm{C}(7)-\mathrm{C}(1)-\mathrm{C}(2)-\mathrm{N}(2)$ & $4.72(15)$ & $\mathrm{C}(6)-\mathrm{C}(1)-\mathrm{C}(7)-\mathrm{N}(1)$ & $177.03(10)$ \\
$\mathrm{C}(6)-\mathrm{C}(1)-\mathrm{C}(2)-\mathrm{C}(3)$ & $0.67(16)$ & $\mathrm{C}(2)-\mathrm{C}(1)-\mathrm{C}(7)-\mathrm{N}(1)$ & $-10.67(15)$ \\
$\mathrm{C}(7)-\mathrm{C}(1)-\mathrm{C}(2)-\mathrm{C}(3)$ & $-171.73(10)$ & $\mathrm{C}(2)-\mathrm{N}(2)-\mathrm{C}(8)-\mathrm{N}(1)$ & $-44.78(13)$ \\
$\mathrm{N}(2)-\mathrm{C}(2)-\mathrm{C}(3)-\mathrm{C}(4)$ & $-178.10(11)$ & $\mathrm{C}(2)-\mathrm{N}(2)-\mathrm{C}(8)-\mathrm{C}(12)$ & $79.13(12)$ \\
\hline
\end{tabular}

表 3 化合物 16 的氢键键长和键角 ${ }^{a}$

Table 3 Hydrogen bond distances and angles for compound 16

\begin{tabular}{ccccc}
\hline $\mathrm{D}-\mathrm{H} \cdots \mathrm{A}$ & $d(\mathrm{D}-\mathrm{H}) / \mathrm{nm}$ & $d(\mathrm{H} \cdots \mathrm{A}) / \mathrm{nm}$ & $d(\mathrm{D} \cdots \mathrm{A}) / \mathrm{nm}$ & $\angle(\mathrm{DHA}) /\left({ }^{\circ}\right)$ \\
\hline $\mathrm{N}(1)-\mathrm{H}(1) \cdots \mathrm{O}(1) \# 1$ & $0.0884(16)$ & $0.2016(17)$ & $0.28929(13)$ & $171.4(14)$ \\
$\mathrm{N}(2)-\mathrm{H}(2) \cdots \mathrm{O}(1) \# 2$ & $0.0888(17)$ & $0.2046(18)$ & $0.29298(13)$ & $173.0(15)$ \\
\hline
\end{tabular}

${ }^{a}$ Symmetry code: \#1: $-x,-y+1,-z+1 ; \# 2 ;-x+1 / 2, y-1 / 2, z$.

表 4 化合物 18 的特征键长和键角

Table 4 Bond lengths and angles for compound 18

\begin{tabular}{rc||cc}
\hline Bond & Length/(nm $\times 10)$ & Bond & Angle $/\left({ }^{\circ}\right)$ \\
\hline $\mathrm{O}(1)-\mathrm{C}(1)$ & $1.353(2)$ & $\mathrm{C}(1)-\mathrm{O}(1)-\mathrm{C}(8)$ & $117.86(14)$ \\
$\mathrm{O}(1)-\mathrm{C}(8)$ & $1.469(2)$ & $\mathrm{C}(3)-\mathrm{N}(1)-\mathrm{C}(8)$ & $118.47(16)$ \\
$\mathrm{O}(2)-\mathrm{C}(1)$ & $1.224(2)$ & $\mathrm{O}(2)-\mathrm{C}(1)-\mathrm{O}(1)$ & $117.29(19)$ \\
$\mathrm{N}(1)-\mathrm{C}(3)$ & $1.375(2)$ & $\mathrm{O}(2)-\mathrm{C}(1)-\mathrm{C}(2)$ & $124.58(19)$ \\
$\mathrm{N}(1)-\mathrm{C}(8)$ & $1.438(2)$ & $\mathrm{O}(1)-\mathrm{C}(1)-\mathrm{C}(2)$ & $117.97(18)$ \\
$\mathrm{C}(1)-\mathrm{C}(2)$ & $1.456(3)$ & $\mathrm{C}(7)-\mathrm{C}(2)-\mathrm{C}(3)$ & $119.67(19)$ \\
$\mathrm{C}(2)-\mathrm{C}(7)$ & $1.397(2)$ & $\mathrm{C}(7)-\mathrm{C}(2)-\mathrm{C}(1)$ & $120.99(18)$ \\
\hline
\end{tabular}

表 5 化合物 18 的特征扭角

Table 5 Angles for compound $\mathbf{1 8}$

\begin{tabular}{cc||cc}
\hline Bond & Angle $/\left(^{\circ}\right)$ & Bond & Angle $/\left({ }^{\circ}\right)$ \\
\hline $\mathrm{C}(8)-\mathrm{O}(1)-\mathrm{C}(1)-\mathrm{O}(2)$ & $165.43(15)$ & $\mathrm{C}(8)-\mathrm{N}(1)-\mathrm{C}(3)-\mathrm{C}(2)$ & $23.6(2)$ \\
$\mathrm{C}(8)-\mathrm{O}(1)-\mathrm{C}(1)-\mathrm{C}(2)$ & $-19.0(2)$ & $\mathrm{C}(7)-\mathrm{C}(2)-\mathrm{C}(3)-\mathrm{N}(1)$ & $-178.31(14)$ \\
$\mathrm{O}(2)-\mathrm{C}(1)-\mathrm{C}(2)-\mathrm{C}(7)$ & $-8.4(3)$ & $\mathrm{C}(1)-\mathrm{C}(2)-\mathrm{C}(3)-\mathrm{N}(1)$ & $9.4(2)$ \\
$\mathrm{O}(1)-\mathrm{C}(1)-\mathrm{C}(2)-\mathrm{C}(7)$ & $176.41(14)$ & $\mathrm{C}(7)-\mathrm{C}(2)-\mathrm{C}(3)-\mathrm{C}(4)$ & $3.9(2)$ \\
$\mathrm{O}(2)-\mathrm{C}(1)-\mathrm{C}(2)-\mathrm{C}(3)$ & $163.84(17)$ & $\mathrm{C}(1)-\mathrm{C}(2)-\mathrm{C}(3)-\mathrm{C}(4)$ & $-168.37(15)$ \\
$\mathrm{O}(1)-\mathrm{C}(1)-\mathrm{C}(2)-\mathrm{C}(3)$ & $-11.4(2)$ & $\mathrm{N}(1)-\mathrm{C}(3)-\mathrm{C}(4)-\mathrm{C}(5)$ & $178.89(16)$ \\
$\mathrm{C}(8)-\mathrm{N}(1)-\mathrm{C}(3)-\mathrm{C}(4)$ & $-158.78(16)$ & $\mathrm{C}(2)-\mathrm{C}(3)-\mathrm{C}(4)-\mathrm{C}(5)$ & $-3.5(2)$ \\
\hline
\end{tabular}

表 6 化合物 18 的氢键键长和键角 ${ }^{a}$

Table 6 Hydrogen bond distances and angles for compound 18

\begin{tabular}{ccccc}
\hline $\mathrm{D}-\mathrm{H} \cdots \mathrm{A}$ & $d(\mathrm{D}-\mathrm{H}) / \mathrm{nm}$ & $d(\mathrm{H} \cdots \mathrm{A}) / \mathrm{nm}$ & $d(\mathrm{D} \cdots \mathrm{A}) / \mathrm{nm}$ & $\angle(\mathrm{DHA}) /\left(^{\circ}\right)$ \\
\hline $\mathrm{N}(1)-\mathrm{HN} \cdots \mathrm{O}(2) \# 1$ & $0.0833(19)$ & $0.212(2)$ & $0.2917(2)$ & $160.3(17)$ \\
\hline
\end{tabular}

${ }^{a}$ Symmetry code: $\# 1: x,-y+1 / 2, z+1 / 2$. 
酩结构的最终残差因子 $R_{1}=0.0410, w R_{2}=0.0996$ 来说, 后者更为合理. 因此, 新的转化产物不可能是噁嗪结构, 而应该是较稳定的喹唑啉酮衍生物.
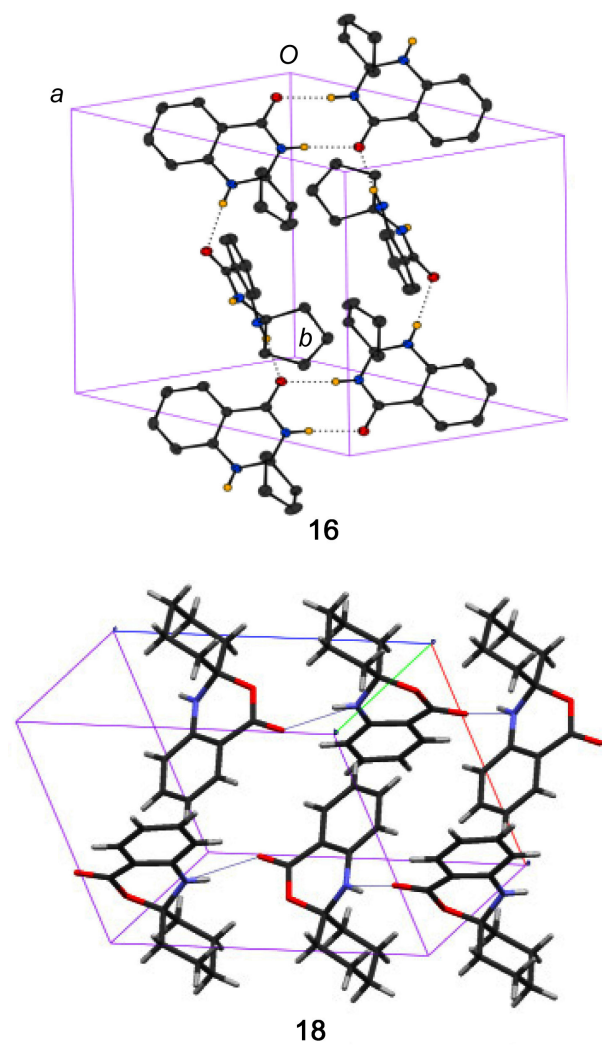

图 9 化合物 16 和 18 的晶胞堆积图

Figure 9 X-ray cell accumulation figure of $\mathbf{1 6}$ and $\mathbf{1 8}$

为了更加直观的对比两种分子的结构, 图 10 给出 了两化合物的晶胞堆积图. 由图 10 可以看出, 喹唑啉酮 衍生物 16 分子堆砌比较密集, 而噁嗪 18 空间堆砌的比 较松散, 两者差异明显.

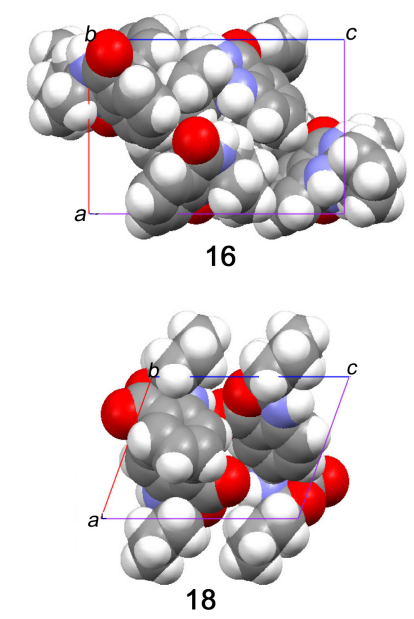

图 10 化合物 16 和 18 的晶胞堆砌图

Figure 10 X-ray cell accumulation figure of $\mathbf{1 6}$ and $\mathbf{1 8}$
Klemm 等 ${ }^{[48]}$ 于 1998 年用邻氨基苯甲酰胺和环戊酮 为原料合成所得的二氢喹唑啉酮 16 并测定了晶体结构. 通过比较 Klemm 等与我们用两种不同途径合成所得同 一产物的晶体结构参数(图 11)可以发现, 它们的结构参 数基本一致. 这说明, 本文建议将新转化产物的骨架结 构确定为二氢喹唑啉酮是十分合理的. 需要指出的是, 两者的结构参数存在着细小差别, 这很可能是测试因素 及相关条件不一致所致(表 7).

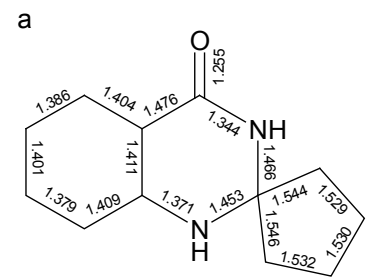

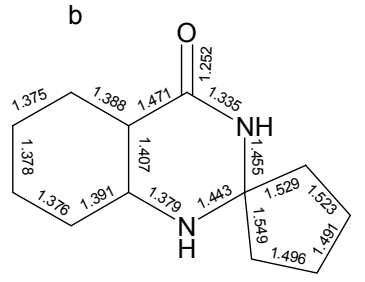

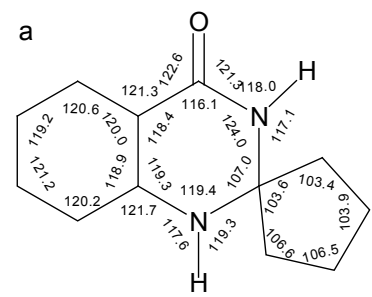

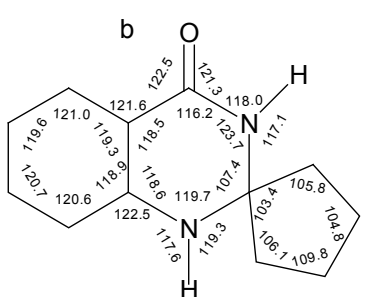

图 11 本课题组(a)和 Klemm 课题组(b)报道的化合物 $\mathbf{1 6}$ 的键 长 $(\mathrm{nm})$ 和键角 $\left(^{\circ}\right)$

Figure 11 Bond lengths $(\mathrm{nm})$ and angles $\left(^{\circ}\right)$ of compound 16 reported by both us and Klemm team

近年来, 一系列新转化产物的单晶已经培养出来, 它们的 X 射线衍射结果都倾向于喹唑啉酮骨架结构(表 8).

综合以上研究, 可以确认邻氨基腈与羰基化合物反 应的新转化产物结构是氢化喹唑啉酮 5 而不是 $2 H-3,1$ 苯并噁嗪(4). 这样不但与波谱表征数据更加符合, 而且 化合物的一些理化性质能够得到更合理的解释.

\section{3 红外光谱}

为了进一步确定新转化产物的结构, 作者将邻氨基 苯甲腈先水解为邻氨基苯甲酰胺 19 , 再用 19 与酮反应 制备了具有确定结构的喹唑啉酮化合物 20(图 12A), 该 物质也可由邻氨基苯甲腈与环已酮直接缩合而成(图 12B). 它们的红外图谱说明了此点(图 13).

\section{4 氢化喹唑啉酮转化的可能机理}

新转化反应的产物结构确定为喹唑啉酮, 其生成也 可能经历苯甲酰胺阶段(图 14). 即邻氨基苯甲腈(1c)在 催化剂作用下发生氧基的水解生成酰胺 19, 然后与酮 
表 7 本课题组和 Klemm 课题组报道的化合物 $\mathbf{1 6}$ 的晶体学参数和结构精修指标

Table 7 Crystallographic data for compound $\mathbf{1 6}$ reported by both us and Klemm team

\begin{tabular}{|c|c|c|}
\hline & Us team & Klemm team \\
\hline Fomula & $\mathrm{C}_{12} \mathrm{H}_{14} \mathrm{~N}_{2} \mathrm{O}$ & $\mathrm{C}_{12} \mathrm{H}_{14} \mathrm{~N}_{2} \mathrm{O}$ \\
\hline Solvent & Ethanol & Methanol \\
\hline Equipment & Rigaku, 2004 & None \\
\hline Temperature/K & $113(2)$ & 294 \\
\hline Wavelength/nm & 0.071070 & 0.071073 \\
\hline Crystal system & Orthorhombic & Orthorhombic \\
\hline Space group & Pbca & Pbca \\
\hline$a / \mathrm{nm}$ & $1.03872(12)$ & $1.0320(2)$ \\
\hline$b / \mathrm{nm}$ & $1.20252(13)$ & $1.1993(3)$ \\
\hline$c / \mathrm{nm}$ & $1.63027(19)$ & $1.6749(3)$ \\
\hline$\alpha, \beta, \gamma$ & $\alpha=\beta=\gamma=90^{\circ}$ & $\alpha=\beta=\gamma=90^{\circ}$ \\
\hline$V / \mathrm{nm}^{3}$ & $2.0363(4)$ & $2.073(2)$ \\
\hline$D_{\mathrm{c}} /\left(\mathrm{g} \cdot \mathrm{cm}^{-3}\right)$ & 1.319 & 1.296 \\
\hline$F(000)$ & 864 & 864 \\
\hline Crystal size $/ \mathrm{mm}^{3}$ & $0.26 \times 0.24 \times 0.16$ & $0.04 \times 0.15 \times 0.43$ \\
\hline Maximum $2 \theta$ & $55.7^{\circ}$ & $50.0^{\circ}$ \\
\hline Reflections collected/Unique/R(int) & $23533 / 2403 / 0.0330$ & $3600 / 1822 /$ no \\
\hline Transmission factor & 0.9864 and 0.9780 & $3600 / 1822 /$ no \\
\hline Data & 2403 & 1141 \\
\hline Refinement parameTers & 142 & 137 \\
\hline$R$ indices $[I>2 \sigma(I)]$ & $R_{1}=0.0410$ & $R_{1}=0.097$ \\
\hline Refine ls $w R$ factor gt $[I>2 \sigma(I)]$ & $w R_{2}=0.0996$ & $w \mathrm{R}_{2}=0.054$ \\
\hline
\end{tabular}

A:<smiles>N#Cc1ccccc1N</smiles>

$1 \mathrm{C}$<smiles>COc1ccc(N)c(C(N)=O)c1</smiles>

19<smiles>O=C1CCCCC1</smiles>
$\underset{p-\mathrm{TsOH}}{\stackrel{\mathrm{DMA}}{\mathrm{p}}}$<smiles>O=C1NC2(CCCC2)Nc2ccccc21</smiles>

20

B:<smiles>N#Cc1ccccc1N</smiles><smiles>O=C1CCCCC1</smiles><smiles>CCCC1(CCC)Nc2cc(C)ccc2C(=N)O1</smiles>

图 12 邻氨基苯甲酰胺及邻氨基苯甲腈与酮的反应

Figure 12 Reaction of cyclohexanone and anthranilamide or/and $o$-aminobenzamide

表 8 已报道的新转化产物晶体结构

Table 8 The structure of new conversion of some quinazolinones reported

Entry Nitrile/amide Carbonyl compd.




\begin{tabular}{|c|c|c|c|c|c|}
\hline Entry & Nitrile/amide & Carbonyl compd. & Structure & X-ray crystallographic & Reference \\
\hline 3 & & & & & {$[61,62]$} \\
\hline 4 & & & & & {$[49,51]$} \\
\hline 5 & & $\mathrm{NO}_{2}$ & & & {$[48,54]$} \\
\hline 6 & & & & & {$[63,64]$} \\
\hline 7 & & & & & [21] \\
\hline 8 & & & & & [20] \\
\hline 9 & & & & & $\begin{array}{l}\text { Our research } \\
\text { without published }\end{array}$ \\
\hline 10 & & & & & [19] \\
\hline 11 & & & & & [22] \\
\hline
\end{tabular}




Entry Nitrile/amide Carbonyl compd.

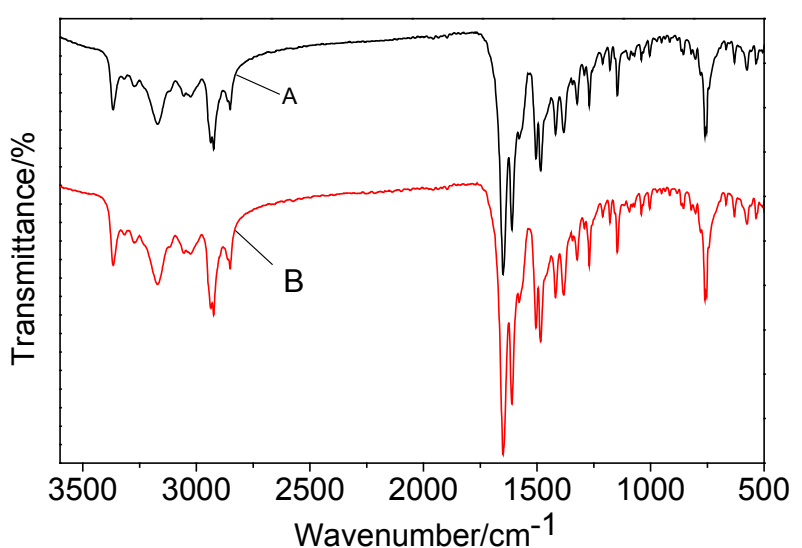

图 13 不同底物反应所得产物的红外对比

Figure 13 The comparison of FT-IR spectra for the product from the different reaction

发生 Michael 反应得到喹唑啉酮 $\mathbf{5 c}$ (路线 A); 也可能不 水解而经由我们提出的路线 $\mathrm{B}^{[22]}$.

\section{1 路线 $A$ 的不合理性}

为了判断转化机理到底是路线 $\mathrm{A}$ 还是路线 $\mathrm{B}$, 作者 使用邻氨基苯甲酰胺与酮为原料, 在路线 B 转化条件下 分别制备了多个喹唑啉酮衍生物, 图 15 中分别列出了 由邻氨基苯甲酰胺与酮反应、邻氨基苯甲腈与酮反应得 到喹唑啉酩衍生物的产率. 由此可以看出, 在 $\mathrm{ZnCl}_{2}$ 催 化下，邻氨基苯甲酰胺与羰基化合物反应的产率明显低 于由邻氨基苯甲腈制备的对应化合物的收率，由此可 见, 这种经过酰胺 19 的反应机理无法解释实验事实. 值 得指出的是, 在已知文献中由邻氨基苯甲酰胺(19)与羰 基化合物反应制备喹唑啉酮时未见使用 $\mathrm{ZnCl}_{2}$ 作催化剂 的报道，一般采用对甲苯磺酸 $(p-\mathrm{TsOH})^{[65 \sim 67]}$.

实验发现，在邻氨基苯甲腈与羰基化合物的反应中 确实有 Schiff碱中间体 $8 \mathrm{c}$ 存在. 对此我们进行了分离并

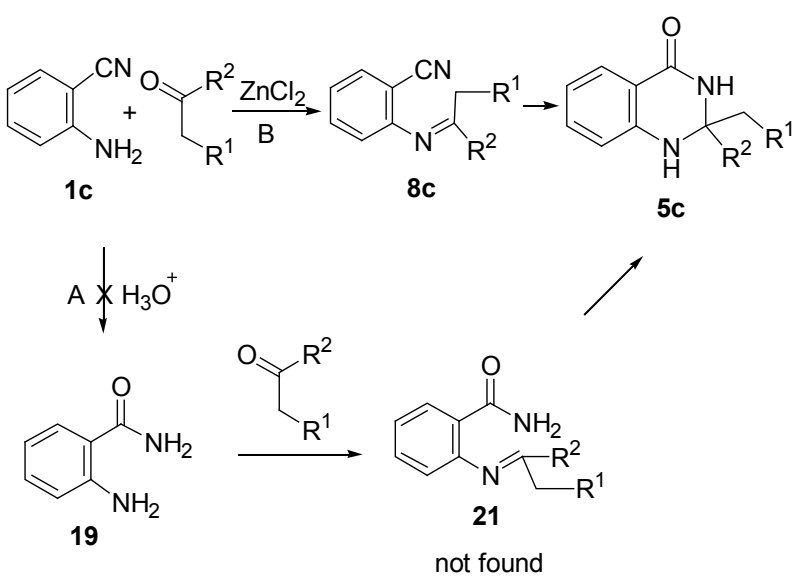

图 14 新转化反应的可能历程

Figure 14 Proposed mechanism of the new conversion

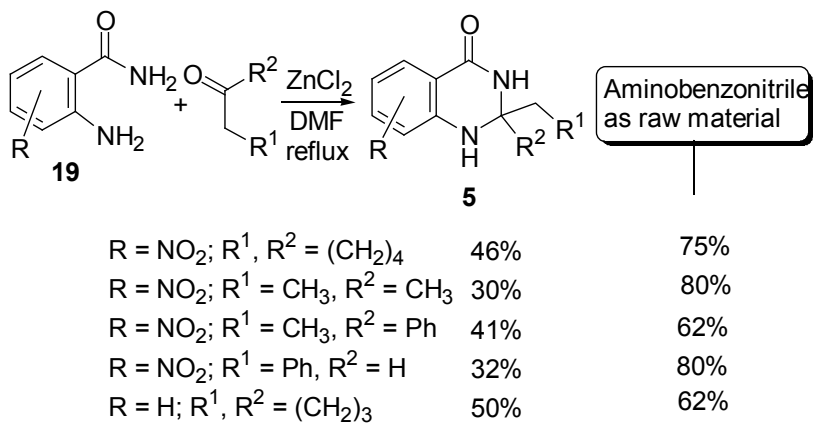

图 15 氯化锌催化下邻氨基苯甲酰胺与酮的反应

Figure 15 Reaction of anthranilamide and cyclohexanone in the catalyst of $\mathrm{ZnCl}_{2}$

获得了此中间产物. 在红外图中明显可见氰基的特征峰 $2224 \mathrm{~cm}^{-1}$ (图 16), 由此可判定邻氨基苯甲腈与酮反应 的中间产物其结构并非 Schiff 碱 21, 而是 Schiff 碱 8c, 因此，这种经由酰胺转化到喹唑啉酮的假设(路线 A) 是 不成立的. 


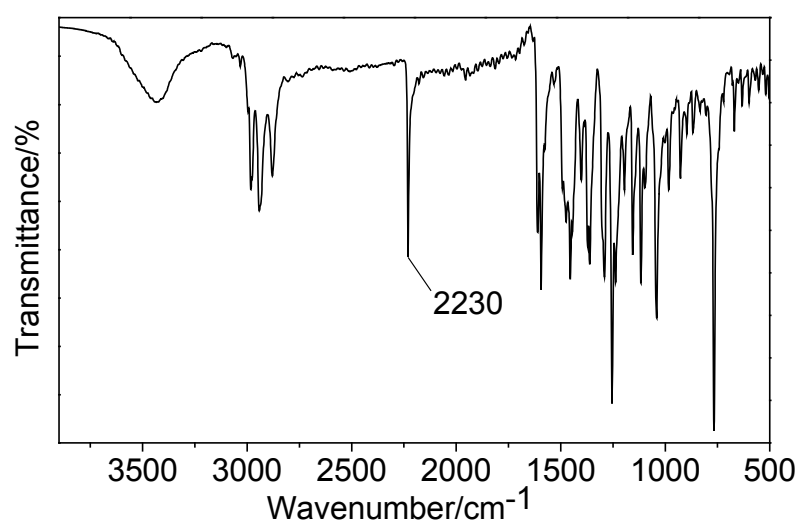

图 16 由邻氨基腈与环酮反应得到的 Schiff 碱 $8 \mathrm{c}$ 的红外光谱

Figure 16 FT-IR of the intermediate $8 \mathrm{c}$

\section{2 如何由噁嗪转化成喹唑啉酮}

根据实验现象和文献资料提出了另一个可能的反 应机理(Scheme 1). 在邻氨基苯甲腈与酮反应时, 芳腈 的氨基进攻酮的羰基碳原子生成氨基加成物后, 差基直 接进攻氧基(Pinner 反应), 得到中间体噁嗪; 由于中间 体噁嗪不稳定, 进一步重排生成喹唑啉酮类产物 (Dimroth 重排反应).

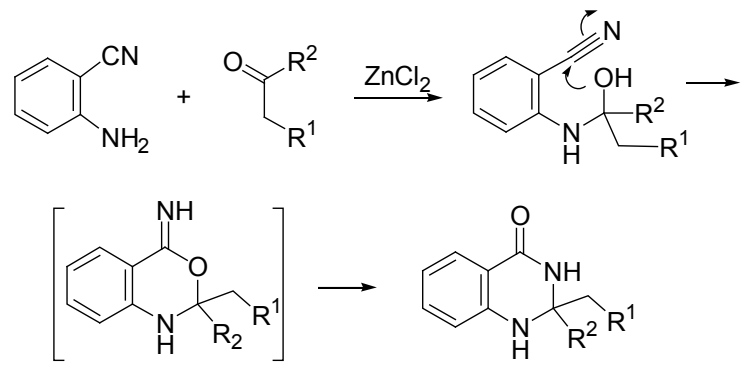

Scheme 1

以上推理是否正确, 关键在于噁嗪衍生物中间体是 否易于生成, 以及生成后是否能够进一步发生转化反 应, 这与反应的动力学和热力学属性有关. 为此, 我们 使用邻氨基苯甲酸(22)与羰基化合物在甲苯中分水回流 (必须保证无水环境), 较快地得到高产率的苯并噁溙-4酮(23)其中也有部分脱水产物 $\mathbf{2 4}$ 生成(Eq. 4). 实验发现, 如果延长反应时间, 则产物 23 逐渐减少, 而 24 的产率 逐渐增加, 这说明噁嗪化合物在热力学上不稳定. 另外, 在后处理阶段, 发现苯并噁嗪䣯 $23 \mathrm{~b}, 23 \mathrm{c}$ 在柱层析以及 重结晶的过程中容易分解, 返回到相应的原料. 其中, 只有 23a 相对稳定一些, 在某些重结晶溶剂中不分解, 但是如果将 23a 在乙醇、水等溶剂或者硅胶中放置, 则 逐渐分解为原料. 而带有强的供电子基团的产物 23c 在 室温下乙酸乙酯中就会逐渐开环分解.

文献中一些相关研究也可以提供这种新转化有力

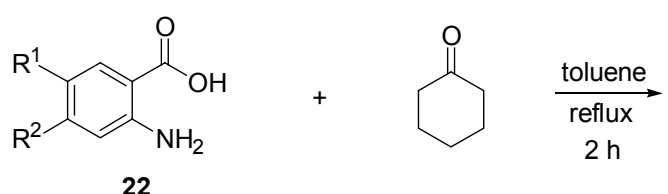

a: $\mathrm{R}^{1}=\mathrm{H}, \mathrm{R}^{2}=\mathrm{H} ; \mathbf{b}: \mathrm{R}^{1}=\mathrm{CH}_{3}, \mathrm{R}^{2}=\mathrm{H}$;

c: $\mathrm{R}^{1}=\mathrm{OCH}_{3}, \mathrm{R}^{2}=\mathrm{OCH}_{3}$

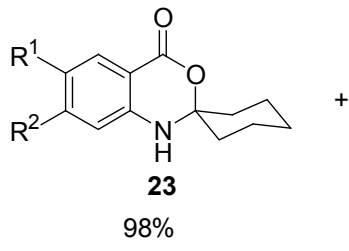

$87 \%$

$89 \%$<smiles>[R]c1cc2[nH]c3c(c(=O)c2cc1[R])CCCC3</smiles>

$<10 \%$
的证据. 图 17 给出了 1999 年 $\mathrm{He}$ 和 Snider ${ }^{[68]}$ 报道的相 关结果. 他们发现, 邻酰胺基酰胺在发生分子内成环反 应时, 并没有按照常规的途径直接由 $\mathrm{N}$ 原子进攻羰基生 成喹唑啉酮，而是先生成了噁嗪中间体，然后重排为喹 唑啉酮结构. 值得注意的是, $\mathrm{He}$ 等研究的亚胺中间体由 于噁嗪环 1,2 -位的双键结构而比较稳定，可以分离出来; 对于本研究的螺环结构, 应该不容易分离得到. 虽然图 17 中的产物与本研究的结构有些差异, 但是至少是一 个相近的例子, 对于本研究提出的机理具有一定的佐证 价值.

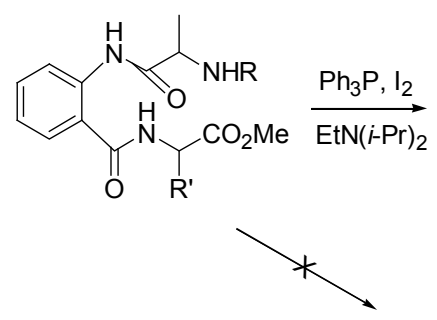<smiles>[R]C1C(=O)NC(C)c2nc3ccccc3c(=O)n21</smiles><smiles>[R]NC(C)c1nc2ccccc2c(=O)n1C([R])COC</smiles>

图 17 邻酰胺基酰胺发生的分子内成环反应

Figure 17 Intramolecular cyclocondensation reaction of anthranilic diamides

由此可见，羟胺中间体的羟基进攻氧基碳发生分子 内的 Pinner 反应 ${ }^{[69]}$, 得到的 1,2-二氢噁嗪酮在热力学上 
较不稳定，进而发生分子内开环到重新闭环的 Dimroth 重排 ${ }^{[70]}$ 生成喹唑啉酮(图 18).

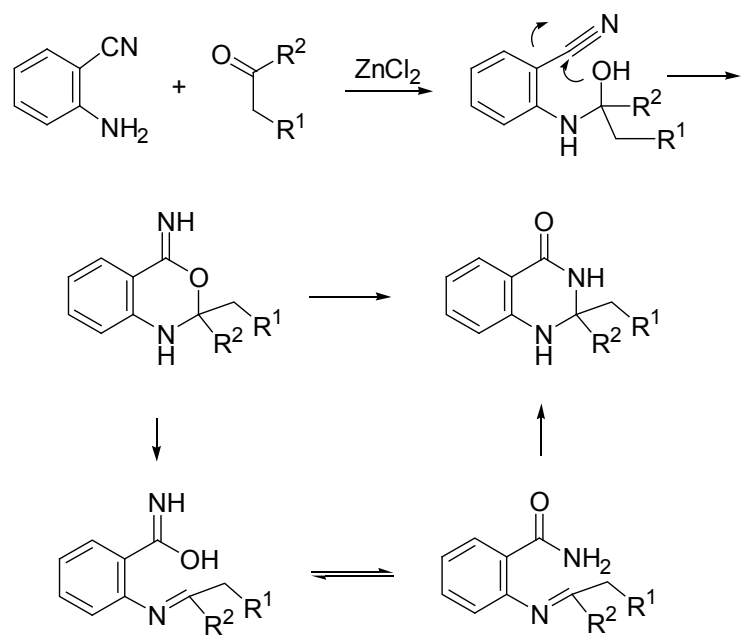

图 18 由噁嗪到喹唑啉酮的 Dimroth 重排

Figure 18 Dimroth from benzoxazine to quinazolinones

Pinner 反应和 Dimroth 重排已是人们十分熟知的有 机人名反应, 两者的组合也在相关研究中得到展示. 如 2006 年, Elkholy 等 ${ }^{[71]}$ 在研究邻氨基氧基吡定与二硫化 碳的反应制备吡啶 $[2,3-d]$ 嘧啶-2,4(1H,3H)-二硫酮时，认 为该反应经历了 Pinner 反应和 Dimroth 重排得到最终产 物(图 19). 2008 年, 瑞士学者 Angelin 等 ${ }^{[72,73]}$ 在研究邻氨 基苯甲醛与硝基乙烷的 Henry 反应时, 发现硝基醇可以 继续发生分子内多米诺 Pinner-Dimroth 反应得到异吲哚 酮类化合物(图 20).

\section{3 邻氨基芳香腈与羰基化合物反应的完整机理}

据此，该类反应的完整机理可以由 Scheme 2 所示. 在邻氨基苯甲腈与酮反应时, 芳腈的氨基进攻酮的羰基 碳原子生成氨基加成物后, 可能沿着两种反应路线进 行：如果沿着 A 路线进行脱水、亲核加成、芳构化即是<smiles>N#Cc1c(N)nc2c(c1-c1ccccc1)CCCC2</smiles><smiles>N=c1sc(=S)[nH]c2nc3c(c(-c4ccccc4)c12)CCCC3</smiles><smiles>S=c1[nH]c(=S)c2c(-c3ccccc3)c3c(nc2[nH]1)CCCC3</smiles>

图 19 氨基氰基吡啶与二硫化碳的反应

Figure 19 Reaction of $\mathrm{CS}_{2}$ and $o$-aminobenzamide pyridine<smiles>N#Cc1ccccc1C=O</smiles><smiles>CCC[N+](=O)[O-]</smiles><smiles>CC(C)C(O)c1ccccc1C#N</smiles><smiles>CC(C)C1OC(=N)c2ccccc21</smiles><smiles>CC(C1NC(=O)c2ccccc21)[N+](=O)[O-]</smiles>

图 20 邻氨基苯甲醛与硝基乙烷的 Henry 反应 Figure 20 Henry reaction of $o$-amino benzaldehyde and nitroethane

经典的 Friedländer 反应, 得到喹啉衍生物; 如果氨基加 成物不脱水，而是沿 $\mathrm{B}$ 路线发生分子内 Pinner 反

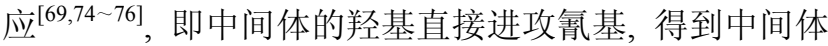
噁嗪，由于中间体噁嗪不稳定，进一步发生 Dimroth 重 排 ${ }^{[70,77,78]}$ 生成喹唑啉酮类产物. 我们 ${ }^{[22]}$ 将该转化命名为 PDF 转化: 即新转化过程首先经过 Pinner 反应生成噁嗪 中间体，而后该中间体进行快速的 Dimroth 重排得到喹 唑啉酮衍生物, 而这一转化过程存在于经典的 Friedländer 反应中.

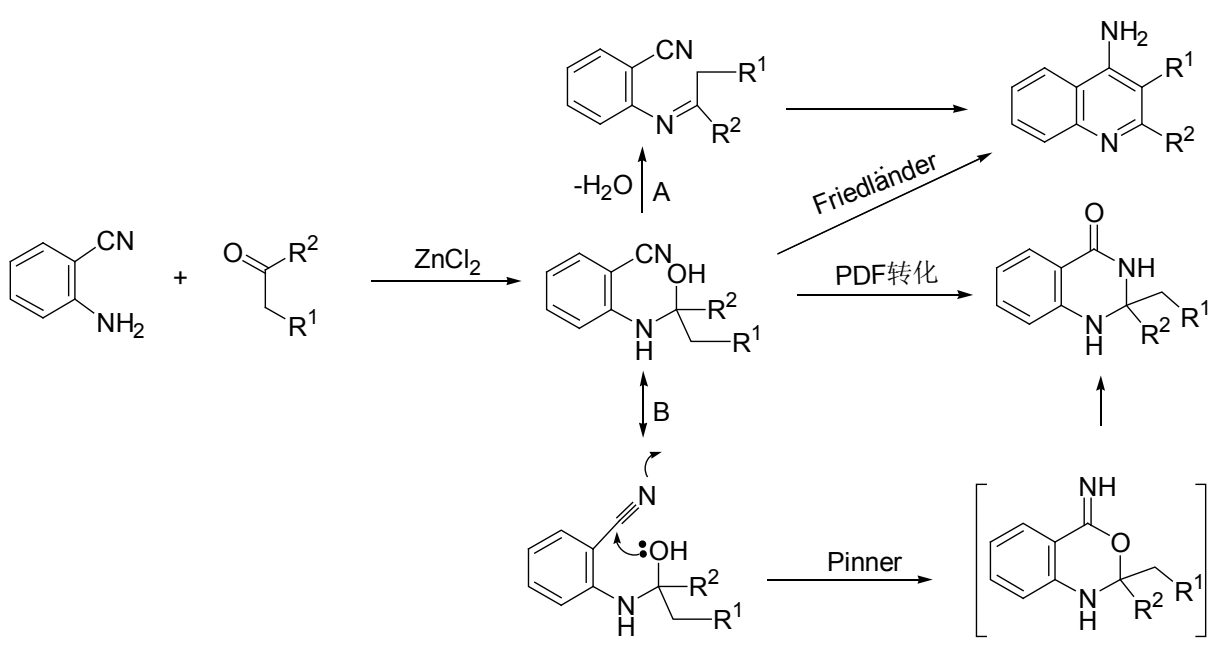

Scheme 2 


\section{5 结论与展望}

通过归纳总结邻氨基苯甲腈与酮发生 PDF 转化所 得产物的 ${ }^{13} \mathrm{C}$ NMR 化学位移和 FT-IR 等光谱数据以及 该产物的 $X$ 射线单晶衍射数据, 确定了产物骨架为喹唑 啉酮结构. 结合邻氨基苯甲腈、邻氨基苯甲酰胺与羰基 化合物的反应现象, 提出 PDF 转化历程为: 氨基加成物 的羟基进攻氧基, 发生 Pinner 反应, 得到噁嗪中间体, 然后该中间体迅速发生 Dimroth 重排生成喹唑啉酮. 实 验结果表明, 噁嗪及喹唑啉酮化合物的稳定性以及邻氨 基苯甲酰胺与羰基化合物的反应现象较好的支持了该 机理.

需要指出的是, 这类反应从发现以来人们只认知了 一半, 只有 PDF 转化的发现才让人们了解了其全貌! 这 也正是有机化学的魅力所在和任务使然, 这种对未知世 界的不断认知和常规认知的渐次突破正是众多有机合
成工作者不知疲倦并孜孜以求的动力所在!

这里, 在鉴定新转化产物结构的反应中我们仅列举 了邻氨基芳香(苯)甲腈与酮这类转化，事实上，后来的 研究表明: 这类 PDF 转化可以在众多不同的底物上获 得应用. 如非芳香邻氨基腈化物与羰基化合物 ${ }^{[22,51]}$ (Scheme 3); 邻羟基腈化物与羰基化合物 ${ }^{[79]}$ (Scheme 4); 包含 PDF 转化的组合转化 ${ }^{[80]}$ (Scheme 5).

同时需要指出的是, 邻氨基腈化物与羰基化合物的 转化是一类非常有意思的新型分岔转化, 由此获得的两 类含氮杂环化合物非常有用 ${ }^{[81,82]}$. Yang ${ }^{[17]}$ 和 $\mathrm{Li}$ 等 ${ }^{[83]}$ 也 已实现获得单一产物的可控分岔转化, 并用 PDF 转化 实施了一系列含氮杂环化合物的简便合成等. 尽管如 此, PDF 转化中的噁嗪中间体是否存在, 能否将新转化 在此停止, 特别是确定机理(含理论计算)和 PDF 产物的 性能、应用等研究有待深入开展下去.

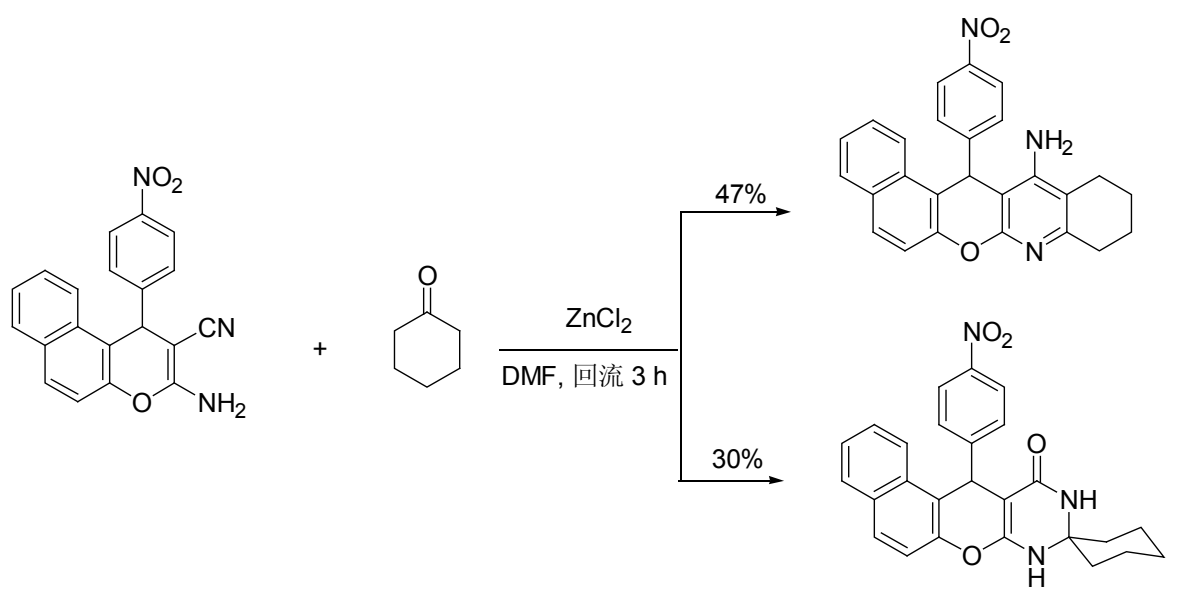

Scheme 3

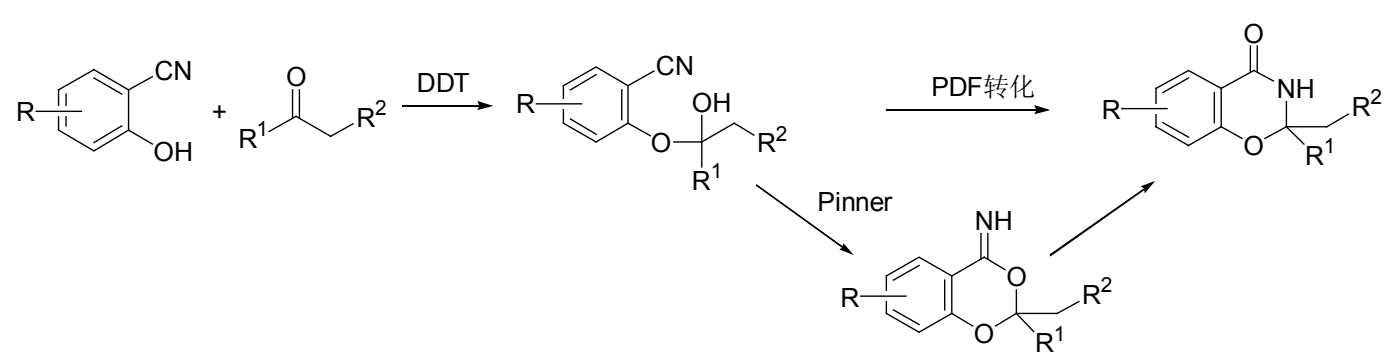

Scheme 4

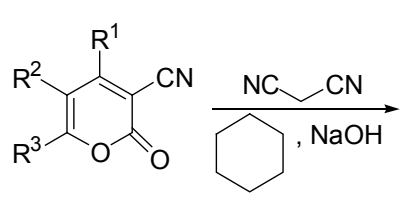

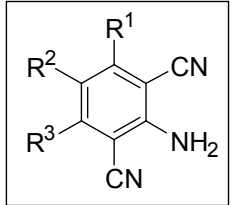

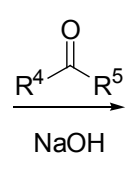<smiles>[R]c1c([R])c(C#N)c2c(c1[R])C(=O)NC([R7])([R])N2</smiles>

Scheme 5 


\section{References}

[1] Richter, M.; Molnár, J.; Hilgeroth, A. J. Med. Chem. 2006, 49, 2838.

[2] Elnagdi, M. H.; Erian, A. W. W. Arch. Pharm. 1991, 324, 853.

[3] Ferris, J. P.; Orgel, L. E. J. Am. Chem. Soc. 1966, 88, 3829.

[4] Dias, A. M.; Cabral, I.; Proença, M. F.; Booth, B. L. J. Org. Chem. 2002, 67, 5546 .

[5] Taylor, E. C.; Wachsen, E. J. Org. Chem. 1978, 43, 4154.

[6] Erian, A. W. Chem. Rev. 1993, 93, 1991.

[7] Abdelrazek, F. M.; Bahbouh, M. S.; Jordan, J. Earth Environ. Sci. 2012, 4, 47 .

[8] Camps, P.; Gómez, E.; Muñoz, Torrero, D.; Badia, A.; Vivas, N. M.; Barril, X.; Orozco, M.; Luque, F. J. J. Med. Chem. 2001, 44, 4733.

[9] McKenna, M. T.; Proctor, G. R.; Young, L. C.; Harvey, A. L. J. Med. Chem. 1997, 40, 3516

[10] Barreiro, E. J.; Camara, C. A.; Verli, H.; Brazil, Mas, L.; Castro, N. G.; Cintra, W. M.; Aracava, Y.; Rodrigues, C. R.; Fraga, C. A. J. Med. Chem. 2003, 46, 1144.

[11] Li, J.-R.; Ma, S.-L. Chin. Chem. Lett. 2005, 16, 1424 (in Chinese). (李加荣, 马淑玲, 中国化学快报, 2005, 16, 1424.)

[12] Zhen, B.; Jiao, Q.; Zhang, Y.; Wu, Q.; Li, H. S.; Shi, D. X.; Li, J. R. Catal. Commun. 2013, 32, 1 .

[13] Yang, L.-P.; Li, J.-R.; Chai, H.-X.; Yuan, H.; Zhang, Q.; Shi, D.-X. Chin. J. Org. Chem. 2013, 33, 174 (in Chinese). (杨留攀, 李加荣, 柴红新, 袁洪, 张奇, 史大昕, 有机化学, 2013, 33, 174.)

[14] Yang, D. L.; Shi, D. X.; Zhang, Q.; Chai, H. X.; Li, J. R. Acta Crystallogr., Sect. B: Struct. Sci. 2013, 69, o633.

[15] Yang, D.-L.; Li, J.-R.; Sun, K.-N.; Lu, H.-Y.; Liu, M.-X.; Shi, D.-X. Chin. J. Org. Chem. 2013, 33, 2341 (in Chinese). (杨德利, 李加荣, 孙克宁, 路红燕, 刘明星, 史大昕, 有机化学, 2013, 33, 2341.)

[16] Yang, L. P.; Shi, D. X.; Chen, S.; Chai, H. X.; Huang, D. F.; Zhang, Q.; Li, J. R. Green Chem. 2012, 14, 945.

[17] Tang, J. H.; Li, J. R.; Zhang, L.; Ma, S. L.; Shi, D. X.; Zhang, Q.; Yang, L. P.; Wang, X.; Liu, X.; Liu, C. J. Heterocycl. Chem. 2012, 49, 533.

[18] Liu, C.-E.; Tang, J.-H.; Li, J.-R. Chin. J. Org. Chem. 2012, 32, 532 (in Chinese).

(刘长娥, 唐建红, 李加荣, 有机化学, 2012, 32, 532.)

[19] Liu, X.; Shi, D. X.; Tang, J. H.; Yang, D. L. Acta Crystallogr., Sect. E: Struct. Sci. 2011, 67, o2016.

[20] Tang, J. H.; Shi, D. X.; Yan, L. P.; Liu, X.; Li, J. R. Acta Crystallogr., Sect. B: Struct. Sci. 2011, 67, o1672.

[21] Li, J. R.; Chen, X.; Shi, D. X.; Ma, S. L.; Li, Q.; Zhang, Q.; Tang, J. H. Org. Lett. 2009, 11, 1193.

[22] Li, J. R.; Zhang, L.; Shi, D. X.; Li, Q.; Wang, D.; Wang, C.; Zhang, Q.; Fan, Y. Synlett 2008, 233.

[23] Wu, X. F.; Oschatz, S.; Block, A.; Spannenberg, A.; Langer, P. Org. Biomol. Chem. 2014, 12, 1865.

[24] Gyuris, M.; Puskas, L. G.; Toth, G. K.; Kanizsai, I. Org. Biomol. Chem. 2013, 11, 6320.

[25] Kanawade, S. B.; Patil, S. P.; Nikam, P. S.; Gangurde, S. A.; Jachak, M. N.; Toche, R. B. J. Heterocycl. Chem. 2012, 49, 363.

[26] Majumdar, K. C.; Roy, B.; Debnath, P.; Taher, A. Curr. Org. Chem. 2010, 14, 846

[27] Marco-Contelles, J.; Pérez-Mayoral, E.; Samadi, A.; Carreiras, M. C.; Soriano, E. Chem. Rev. 2009, 109, 2652.

[28] Shaban, M. E.; Youssef, A. M.; Elaasar, N. K.; EI-Ziaty, A. K. Pak J. Sci. Ind. Res. 2008, 51, 119.

[29] Kouznetsov, V. V.; Mendez, L. Y.; Gomez, C. M. Curr. Org. Chem. $\mathbf{2 0 0 5}, 9,141$

[30] Riesgo, E. C.; Jin, X.; Thummel, R. P. J. Org. Chem. 1996, 61, 3017.

[31] Yang, D.; Jiang, K.; Li, J.; Xu, F. Tetrahedron 2007, 63, 7654.

[32] Palimkar, S. S.; Siddiqui, S. A.; Daniel, T.; Lahoti, R. J.; Srinivasan, K. V. J. Org. Chem. 2003, 68, 9371.

[33] Dormer, P. G.; Eng, K. K.; Farr, R. N.; Humphrey, G. R.; McWilliams, J. C.; Reider, P.; Sager, J. W.; Volante, R. J. Org. Chem. 2003, 68, 467.

[34] Cheng, C. C.; Yan, S. J. Org. React. 1982, 28.

[35] Muchowski, J. M.; Maddox, M. L. Can. J. Chem. 2004, 82, 461.

[36] Leon, R.; Garcia, A. G.; Marco-Contelles, J. J. Chem. Res. 2006, 8, 536.

[37] Rane, B. S.; Kazi, M. A.; Bagul, S. M.; Shelar, D. P.; Toche, R. B.; Jachak, M. N. J. Fluoresc. 2010, 20, 415.

[38] Perez-Mayoral, E.; Musilova, Z.; Gil, B.; Marszalek, B.; Polozij, M.; Nachtigall, P.; Cejka, J. Dalton Trans. 2012, 41, 4036.

[39] Zhang, L. J.; Yu, J. L.; Wang, W. L.; Li, H.; Xu, D. D.; Bi, Y. D.; Liu, F. D. Tetrahedron Lett. 2014, 55, 710.

[40] Safari, J.; Gandomi-Ravandi, S. C. R. Chim. 2013, 16, 1158.

[41] Nguyen, T. B.; Ermolenko, L.; Al-Mourabit, A. Green Chem. 2013, $15,2713$.

[42] Labade, V. B.; Shinde, P. V.; Shingare, M. S. Tetrahedron Lett. 2013, 54, 5778.

[43] Desroses, M.; Scobie, M.; Helleday, T. New J. Chem. 2013, 37, 3595 .

[44] Sharma, R.; Pandey, A. K.; Chauhan, P. M. S. Synlett 2012, 23, 2209.

[45] Ghashang, M. Orient. J. Chem. 2012, 28, 1213.

[46] Spagnol, G.; Rajca, A.; Rajca, S. J. Org. Chem. 2007, 72, 1867.

[47] Bonne, D.; Dekhane, M.; Zhu, J. Org. Lett. 2005, 7, 5285.

[48] Klemm, L. H.; Weakley, T. J. R.; Gilbertson, R. D.; Song, Y. H. J. Heterocycl. Chem. 1998, 35, 1269

[49] Zhang, L. J.; Li, J. R.; Shi, D. X.; Chen, J. Acta Crystallogr., Sect. E: Struct. Sci. 2008, 64, o449.

[50] Zhang. L.; Shi, D. X.; Li, J. R.; Zhang, L.; Fan, Y. Q. Acta Crystallogr., Sect. E: Struct. Sci. 2008, 64, o1056.

[51] Zhang, L.; Shi, D. X.; Fan, Y. Q.; Qian, D.; Li, J. R. Acta Crystallogr., Sect. E: Struct. Sci. 2009, 65, o1345

[52] Zhang, L.; Li, J. R.; Yang, X.; Shi, D. X.; Chen, J. Acta Crystallogr., Sect. E: Struct. Sci. 2008, 64, o450.

[53] Zhang, L. J.; Li, J. R.; Shi, D. X.; Zhang, L.; Fan, Y. Q. Acta Crystallogr., Sect. E: Struct. Sci. 2008, 64, o448.

[54] Li, J.-R.; Zhang, L.-J.; Shi, D.-X.; Zhang, Q. Chin. Chem. Lett. 2008, 19, 15 (in Chinese). (李加荣, 张立军, 史大昕, 张奇, 中国化学快报, 2008, 19, 15.)

[55] Chao, X.; He, X.; Yang, Y.; Zhou, X.; Jin, M.; Liu, S.; Cheng, Z.; Liu, P.; Wang, Y.; Yu, J.; Tan, Y.; Huang, Y.; Qin, J.; Rapposelli, S.; Pi, R. Bioorg. Med. Chem. Lett. 2012, 22, 6498.

[56] Gutschow, M.; Neumann, U.; Sieler, J.; Eger, K. Pharm. Acta Helv. 1998, 73, 95

[57] Bernstein, J.; Davis, R. E.; Shimoni, L.; Chang, N. L. Angew. Chem., Int. Ed. 1995, 34, 1555.

[58] Etter, M. C.; MacDonald, J. C.; Bernstein, J. Acta Crystallogr., Sect. E: Struct. Sci. 1990, 46, 256.

[59] Li, J. R.; Ma, S. L.; Sun, Y. J.; Wei, X. J.; Zhou, Z. M. J. Heterocycl. Chem. 2006, 43, 745 .

[60] Li, J.-R.; Sun, Y.-J.; Zhou, Z.-M. Chin. J. Org. Chem. 2006, 26, 928 (in Chinese) (李加荣，马淑玲，孙永江，周智明，有机化学, 2006, 26, 928.)

[61] Shi, D.; Dou, G.; Zhou, Y. Synthesis-Stuttgart 2008, 13, 2000.

[62] Shi, D. Q.; Rong, L. C.; Wang, J. X.; Zhuang, Q. Y.; Wang, X. S.; 
Hua, H. W. Tetrahedron Lett. 2003, 44, 3199.

[63] Cheng, X.; Vellalath, S.; Goddard, R.; List, B. J. Am. Chem. Soc. 2008, 130, 15786.

[64] Chinigo, G. M.; Paige, M.; Grindrod, S.; Hamel, E.; Dakshanamurthy, S.; Chruszcz, M.; Minor, W.; Brown, M. L. J. Med. Chem. 2008, 51, 4620.

[65] Diwu, Z.; Lu, Y.; Upson, R. H.; Zhou, M.; Klaubert, D. H.; Haugland, R. P. Tetrahedron 1997, 53, 7159.

[66] Zappalà, M.; Grasso, S.; Micale, N.; Zuccalà, G.; Menniti, F. S.; Ferreri, G.; De, S. G.; De, M. C. Bioorg. Med. Chem. Lett. 2003, 13, 4427.

[67] Mhaske, S. B.; Argade, N. P. Tetrahedron 2006, 62, 9787.

[68] He, F.; Snider, B. B. J. Org. Chem. 1999, 64, 1397.

[69] Siskos, A. P.; Hill, A. M. Tetrahedron Lett. 2003, 44, 789.

[70] Zaki, M. Molecules 1998, 3, 71.

[71] Elkholy, Y. M.; Morsy, M. A. Molecules 2006, 11, 890.

[72] Angelin, M.; Vongvilai, P.; Fischer, A.; Ramström, O. Chem. Commun. 2008, 6, 768.

[73] Angelin, M.; Fischer, A.; Ramström, O. J. Org. Chem. 2008, 73, 3593.

[74] Lajoie, G.; Lepine, F.; Maziak, L.; Belleau, B. Tetrahedron Lett. 1983, 24, 3815.
[75] Neugebauer, W.; Pinet, E.; Kim, M.; Carey, P. R Can. J. Chem. 1996, $74,341$.

[76] Luzyanin, K. V.; Kukushkin, V. Y.; Kuznetsov, M. L.; Garnovskii, D. A.; Haukka, M.; Pombeiro, A. J. Inorg. Chem. 2002, 41, 2981.

[77] Wang, J.; Wang, J.; Zhu, Y.; Lu, P.; Wang, Y. Chem. Commun. 2011, $47,3275$.

[78] Lin, G.-C.; Liu, L.; Zhang, L.- R.; Zhang, L.-H. Chin. J. Inorg. Chem. 2002, 10, 405 (in Chinese). (林桂椿, 刘莉, 张亮仁, 张礼和, 合成化学, 2002, 10, 405.)

[79] Kurz, T.; Widyan, K.; Wackendorff, C.; Schlüter, K. Synthesis 2004, 1987.

[80] Liu, X.; Shi, D.-X.; Tang, J.-H.; Zhang, Q.; Li, J.-R. Chin. J. Org. Chem. 2011, 31, 1710 (in Chinese).

(刘璇, 史大昕, 唐建红, 张奇, 李加荣, 有机化学, 2011, 31, 1710.)

[81] Zhang, P. W.; Terefenko, E. A.; Fensome, A.; Wrobel, J.; Winneker, R.; Lundeen, S.; Marschke, K. B.; Zhang, Z. M. J. Med. Chem. 2002, 45, 4379.

[82] Kamal, A.; Ramana, K. V.; Rao, M. V. J. Org. Chem. 2001, 66, 997.

[83] Yang, L. P.; Li, J. R.; Chai, H. X.; Lu, H. Y.; Zhang, Q.; Shi, D. X. Chin. J. Chem. 2013, 31, 443.

(Qin, X.) 